\title{
Ocular Leprosy in Iran: Findings of a Random Survey at the Baba Baghi Leprosarium, Tabriz*
}

\author{
K. RAMANUJAM, $†$ P. R. SUNDAR AND A. A. KHAMNEI \\ Baba Baghi Leprosarium, Tabriz, Iran
}

\begin{abstract}
Ocular manifestations in 100 cases of leprosy, mostly lepromatous, in a racial group susceptible to serious leprosy are presented. The pathogenesis of the eye involvement is discussed. Its prevention and management are recounted solely with a view to emphasize the fact that the institution of simple procedures will go a long way in calling a halt to the ocular tragedy in leprosy.
\end{abstract}

"To lose one's eye sight when the sense of touch has already gone is a double disaster and it requires only a little imagination to appreciate the magnitude of such calamity."

Walter Fancott

\section{Introduction}

Visual impairment, sometimes culminating in total loss of vision is a major tragedy in the life of a patient with leprosy. Deformities, disabilities and neuropathic ulceration in the peripheral parts of the extremities sometimes lead to dehabilitation of the patients, thereby entailing loss of self-support, man power and sometimes even self respect. Such a situation can, however, be stemmed and even retrieved by instituting appropriate preventive and corrective measures, and the patient returned to society as a self-reliant and useful citizen. In sharp contrast, involvement of the eyes with the consequent impairment or loss of vision in the course of leprosy infection renders the patient utterly helpless and absolutely dependent. The fact that this visual disability or loss of vision is to a large extent preventable, if only certain simple and appropriate measures had been taken in time, adds poignancy to the situation.

When we started work at the Baba Baghi Leprosarium in 1975, one of the things that arrested our attention was the unusually large number of patients with facial nerve damage manif esting itself often as a total facial paralysis. The frequency and severity of damage to the unprotected eye consequent on the

Received for publication 3 March 1978.

* Based on a paper presented at the Seminar on "Evaluation of leprosy", held in Teheran, Iran, in June 1976.

† Present address: Specialist Leprologist, Schieffelin Leprosy Research \& Training Centre, K arigiri, N.A. Dist. Tamilnadu-632106.

0305-7518/78/0901-0231\$01.00/0 - 1978 British Leprosy Relief Association 
nerve paralysis resulting in various grades of visual loss was also striking. Damage to the facial nerve does occur in leprosy, but the frequency of such damage encountered here was out of all proportion to that seen in India. It was the experience of one of us (K. R.) that in India the involvement of the facial nerve in leprosy was very infrequent and that this more often manifested itself as an isolated paralysis of its zygomatic branch rather than that of the entire nerve. In the Baba Baghi Leprosarium the visual damage sustained by some of the patients in all types of leprosy consequent on the loss of the protective mechanism of the eye and its subsequent neglect was supplemented by the occurrence of chronic plastic iridicyclitis in the lepromatous cases. There were a few instances where both these factors were in operation in the same subject. In view of the magnitude and severity of the the ocular involvement it was felt worthwhile to undertake a random survey of the ocular damage caused by leprosy in this institution with a view to determine its frequency and nature.

\section{Material and Methods}

Our random survey of the ocular involvement was confined to the cases admitted into the wards of the Baba Baghi Leprosarium for the treatment of the complications of leprosy or intercurrent illnesses and also to the patients attending the outpatient department for advice. The patients were first subjected to a clinical examination supplemented with the examination of skin smears in order to classify the cases. This was followed by examination of the face in general, and of the eyes and their adnexa in particular. The findings were recorded on a suitably drawn proforma which included the biodata of the patient, the clinical classification of the case, the presence of paralysis or paresis of the muscles of the face, sensory status of the face and the cornea, presence of madarosis and the condition of the eyes in respect of the sclera, cornea, iris and the pupil. The elicitation of corneal sensation in some of the subjects was found to be very difficult because of the total paralysis of the eyelid muscles. The examination of the eye was carried out with a hand-torch and binocular loupe.

None of us was a qualified ophthalmologist, but owing to long association with the speciality (leprosy) and by force of circumstances, a working knowledge of the recognition and management of the ocular involvement in leprosy had been acquired. This is our apology for any shortcomings or misinterpretation of findings in this paper.

The survey under report covers the findings in 100 patients, comprising 80 lepromatous, 8 borderline and 12 indeterminate cases examined at random. In some of these subjects who appeared to have been quiescent for years ('burntout' cases) the classification of the disease was made from the residual signs or stigmata of the disease when present and occasionally by surmise.

\section{Findings}

The findings of the study are briefly as follows:

(1) The involvement of the seventh cranial nerve manifesting as total facial paralysis or isolated paralysis of the zygomatic branch was detected in $49.0 \%$ 
of the cases examined, the former accounting for $26.0 \%$ and the latter for $23.0 \%$. These patients exhibited varying grades of visual defect as a result of the damage sustained by the cornea in the unprotected eye. This manifested itself as anything varying from nebula and leucoma to complete replacement of the cornea by dense scar tissue (Figs 1 and 2). This type of ocular involvement occured in all types of leprosy. The taste sensation on the anterior two-thirds of the tongue could not be tested in these subjects owing to the difficulty in communicating with each other. This is mentioned particularly because one of

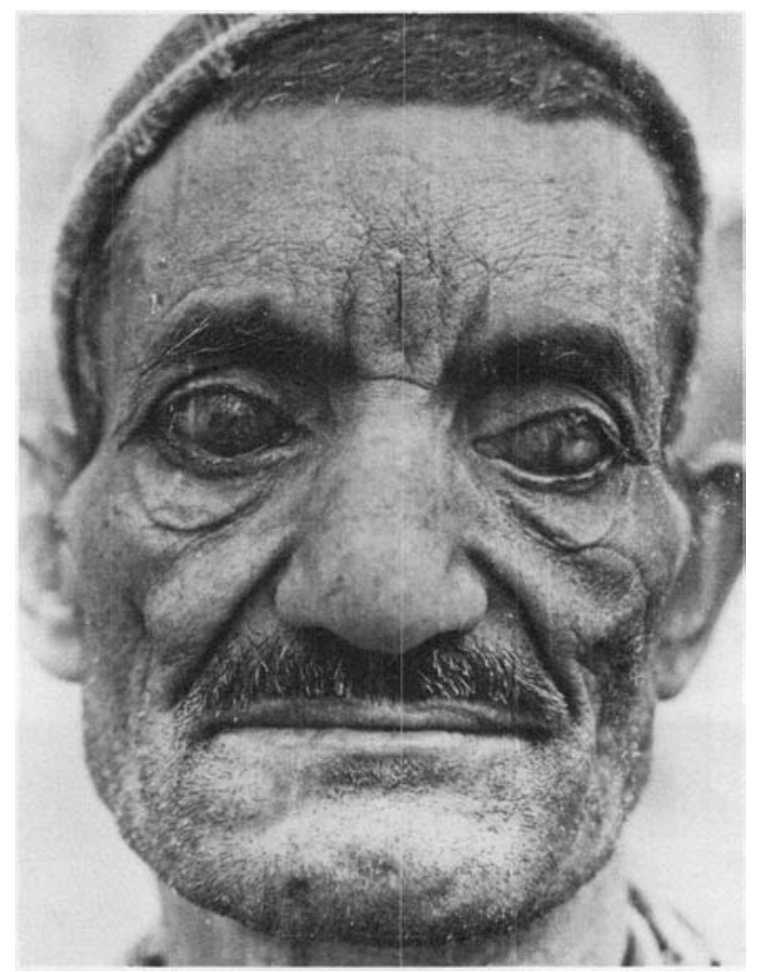

Fig. 1. Bilateral lagophthalmos with exposure keratitis.

us (K. R.) while working at the Central Leprosy Teaching Research Institute, Chengalpattu, India, detected the loss of taste over the anterior two-thirds of the tongue in 2 patients who manifested isolated paralysis of the zygomatic branch.

(2) The involvement of the elements in the anterior segment of the eye with its protean manifestations consequent on bacillary invasion was observed in the multibacillary types of leprosy, especially lepromatous cases. One of the surprising findings was the presence of signs of chronic plastic iridocyclitis in 2 cases clinically labelled as indeterminate leprosy. The manifestations in the 


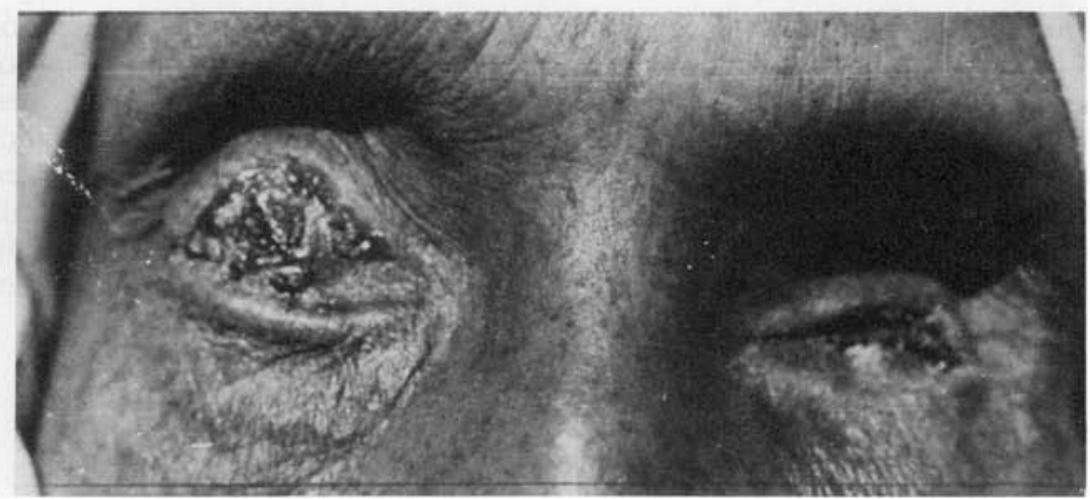

Fig. 2. Replacement of the cornea by dense scar tissue.

order of their frequency were: chronic plastic iridocyclitis with its sequelae, sclerosing keratitis, subscleral nodule, corneal and ciliary staphyloma. Iris pearls or lepromata were not detected in any of the cases by the procedure adopted. Cataract, not an infrequent complication in lepromatous cases in India, was seen in 4 subjects. What appeared to be a widely dilated pupil, but in reality the result of iris atrophy, was seen in 2 cases, either on one or both sides. Anaesthesia of the cornea was detected in 5 cases. There were 2 cases each of dacryocystitis (Fig. 3) and phthisis bulbi.

Chronic plastic iridocyclitis of varying grades of severity was observed in $76.0 \%$ of lepromatous cases, 2 of the 8 cases of regressed borderline leprosy and 2 cases of indeterminate leprosy. In most of the instances, the iris with a

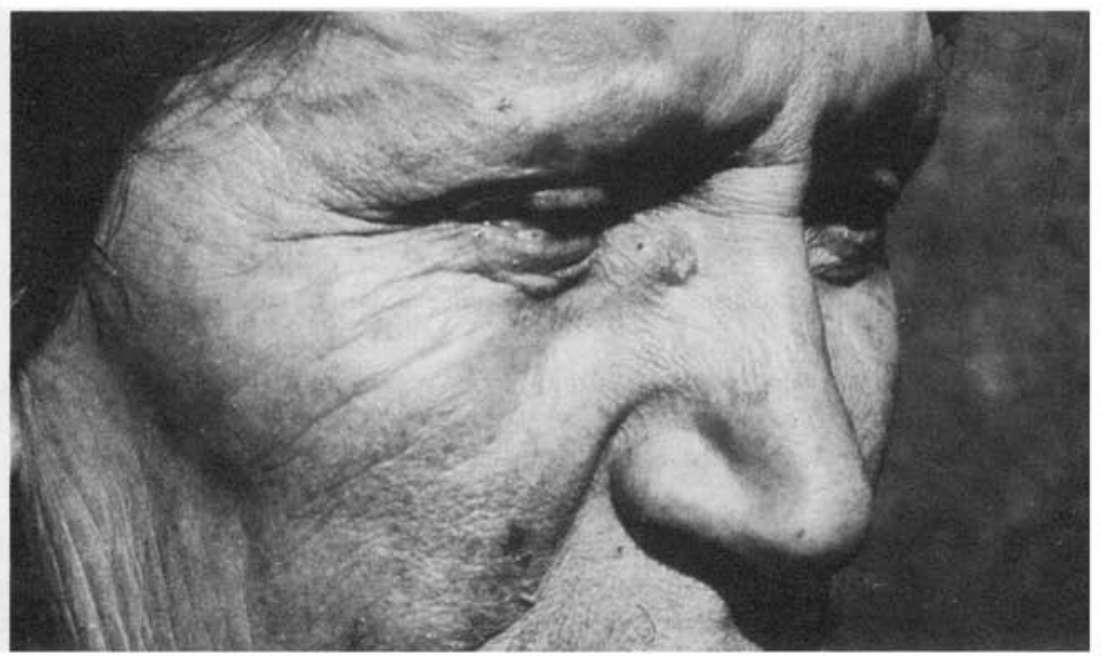

Fig. 3. Dacryocystitis. 
small irregular pupil was firmly bound down by posterior synechiae. In one case the pupillary aperture was plugged with exudate (Fig. 4). Atrophic changes in the iris varied from localized areas of loss of pigment leaving behind the stroma of the iris to total loss of iris substance leading to a "moth-eaten" appearance and coloboma of the iris (Figs 5 and 6). In one case this led to a condition of polycoria (Fig. 7).

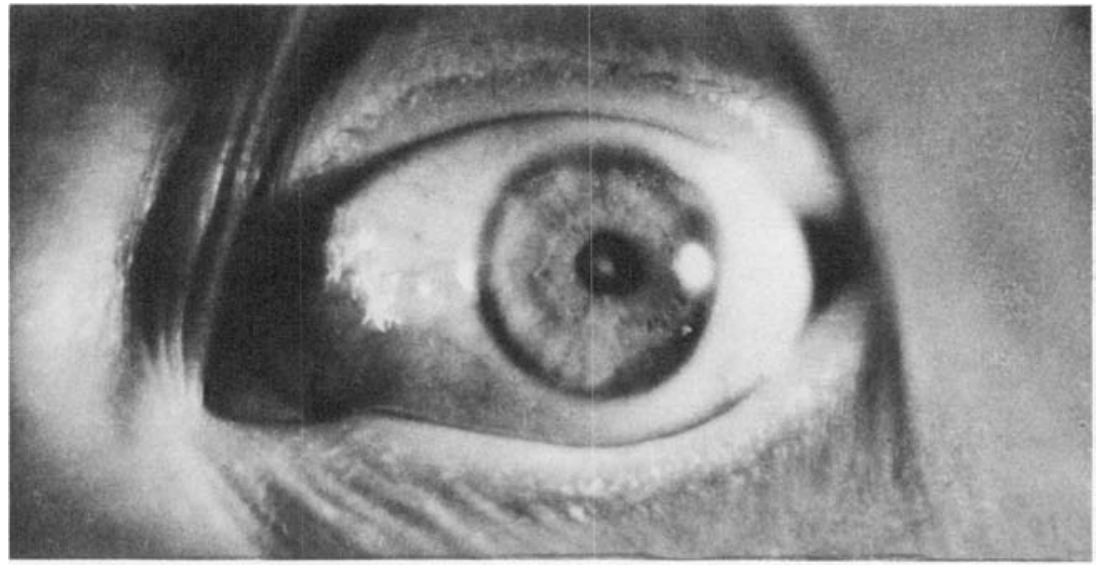

Fig. 4. Exudate in the pupillary aperture.

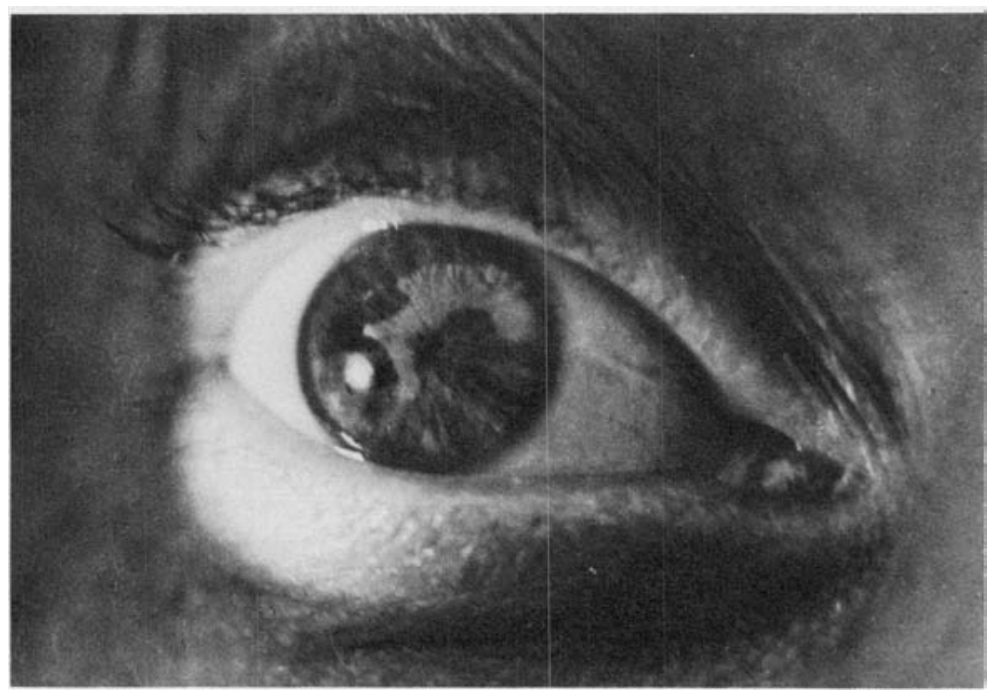

Fig. 5. Atrophy of the iris-"moth-eaten" appearance of the iris. 


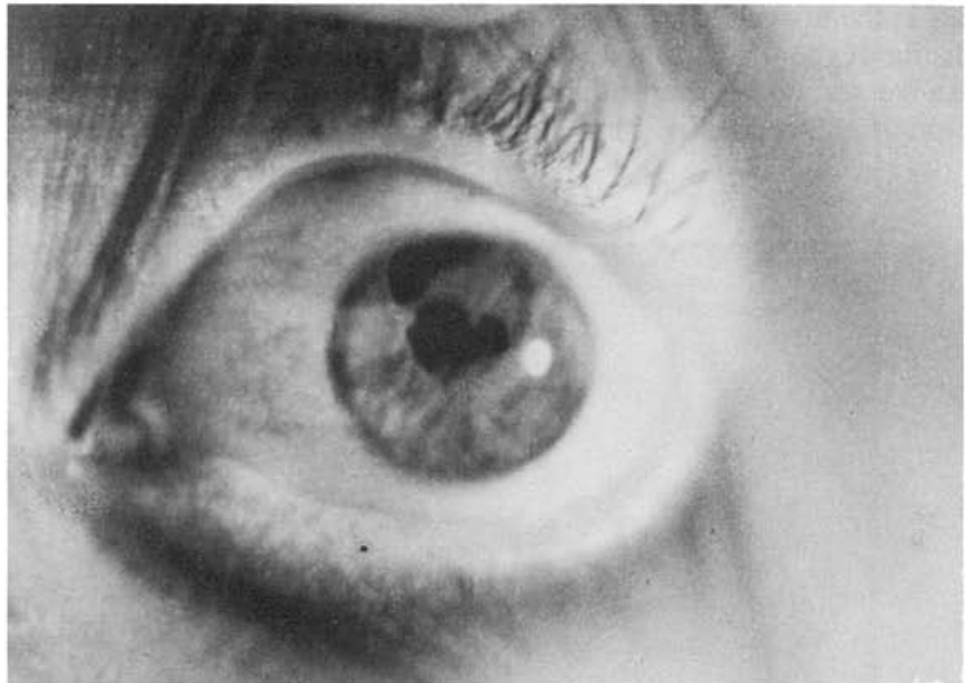

Fig. 6. Atrophy of the iris, leading to loss of iris stroma - coloboma of the iris.

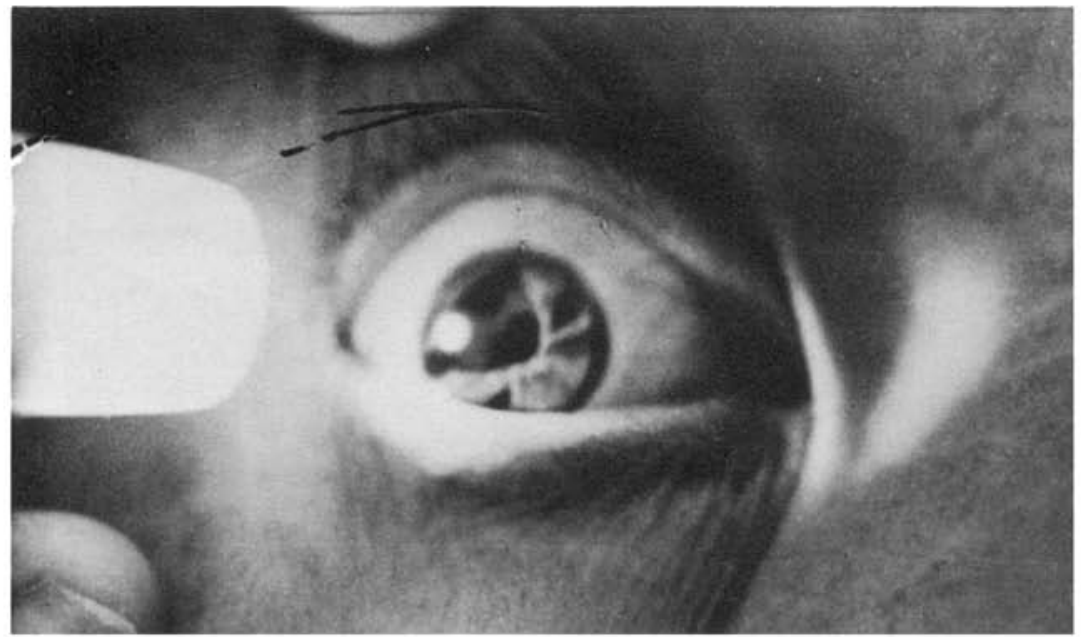

Fig. 7. Atrophy of the iris leading to "polycoria". 


\section{Discussion}

Ocular involvement in leprosy occurs in one of 2 ways: (i) from mechanical causes and here the discussion will be limited to the damage arising from the loss of "blink reflex", the protective mechanism of the eye, following damage to the zygomatic branch of the facial nerve, and (ii) actual invasion of the elements in the anterior segment of the eye by Mycobacterium leprae in the lepromatous and near lepromatous cases producing, among other things, an insidious, painless and relentlessly progressive destruction of the iris and the ciliary body.

Facial nerve involvement in leprosy is encountered in all types of the disease with perhaps greater frequency in the borderline and tuberculoid cases. One of the peculiarities of this nerve involvement in this disease is the great tendency for the occurrence of an isolated paralysis of one of the branches rather than of the nerve trunk itself. The branch of predilection appears to be the zygomatic branch. In India, Dewan (quoted by Antia, 1966) observed facial nerve involvement in $3.0 \%$ of cases and that this often manifested itself as lagophthalmos. Abraham (1976) observed lagophthalmos in $2.0 \%$ of cases. The lower branches of the facial nerve are said to be more involved in the yellow and white skinned races (Antia et al., 1966a). Attempts have been made to investigate the cause of facial nerve damage in leprosy. Brand's hypothesis (1959) that $M$. leprae prefers the colder parts of the body and the nerves for its localization and destructive work would perhaps explain the damage to the zygomatic branch with greater frequency. Antia et al. (1966b) by extensive operative exploration of the facial nerve in the side of the face confirmed the clinical impression that the zygomatic branch was the one most frequently affected. They also observed that the greater frequency of involvement of the distal part of the nerve to the orbicularis oculi muscle "possibly arose from the anastomosing sensory branches of the maxillary nerve". Secondary factors such as "the apposition of this nerve against the unyielding bony arch of the zygoma and its possible compression there, increased fibrosis and fibrous tunnels sleeving these branches", together with exposure to cold, and trauma, all may play a part in the picking out of this branch with greater frequency. From the observations made on the Baba Baghi subjects in Tabriz where during the winter months there is very heavy snowfall and the temperature goes well below zero, it would appear that by far the more significant factor operating in the causation of paralysis of the facial nerve could be the effect of cold temperature on the superficially located parts of the nerve which have been conditioned for damage by the invasion and inflammation set up by $M$. leprae. If this were so, the frequency of such involvement should be more in the winter months. This needs elucidation. Another factor operating in these subjects is the unchecked progress of the disease in the absence of specific treatment. In addition to the above, inflammatory infiltration into the nerve and spasm of the vasa nervora leading to ischaemia of the nerve and thereby interf erence with its function may be the other factors operating in these cases.

The manner and nature of involvement of the elements in the anterior segment of the eye by bacillary invasion in the multibacillary types of leprosy 
are too well known to merit repetition here. It may however be mentioned that the frequency and severity of ocular involvement in these cases is directly proportional to the duration and degree of advancement of the disease, the ethnic background and treatment status of the patient. Harrell (1977) in his survey of "ocular leprosy in the Canal zone" observed a high prevalence of leprotic ocular disease $(96.0 \%)$ amongst 48 patients, mostly lepromatous, and that $64.0 \%$ gave evidence of present or past iritis". He believes that this high prevalence "is most probably due to the advanced age of the patients, the lengthy duration of their illness and the high percentage of persons affected by the lepromatous form of the disease". Gupta (1976) in a report based on the examination of 300 cases of leprosy, mostly lepromatous, at the Central Leprosy Teaching and Research Institute, Chengalpattu, found that: (i) the incidence of ocular complications was $69.0 \%$ (nature not mentioned), (ii) $50.0 \%$ of the patients without ocular involvement were young subjects (less than 20 years old) suggesting that the ocular complications were most frequent with the increasing duration of the disease and (iii) the incidence of blindness was low, $3.0 \%$ resulting from cataract, $2.0 \%$ from corneal opacities and $0.66 \%$ due to iridocyclitis. This extremely low prevalence of chronic plastic iridocyclitis leading to loss of vision is presumably due to access of the Sanatorium population to specific treatment and also to their ethnic background. In sharp contrast, the involvement of the iris and the ciliary body in the Baba Baghi cases is very high and the destructive effect of this involvement is also striking. This, we believe, is largely due to the neglect of specific treatment and also to their racial extraction. The atrophic changes observed in the iris in some of the cases and which Brand (1976) calls "progressive iris atrophy" are said to be the consequence of "prolonged perhaps low grade iris inflammation". She believes that this condition contributes to the development of a low grade glaucoma "arising from the silting of the pigment lost from the iris against the trabeculae at the anterior angle, thereby obstructing the outflow of aqueous".

\section{Management}

"Foresight prevents blindness" was the theme with which the World Health Day April 1976, "put the spot light on the darkness which envelopes so many" (World Health, 1976). In leprosy the institution of certain simple preventive and therapeutic measures will go a long way in averting a calamitous situation in both the paralytic and Mycobacterial involvement of the eye. These methods are well known but merit repetition here, by way of emphasis.

In the paralytic type of eye involvement the eye should be protected from glare and dust during the waking hours by the use of an eye shade. Under rural conditions this can be achieved by teaching the patient to tie his turban cloth or any piece of clean cloth across the affected eye. Dark glasses with sideprotectors will achieve the same purpose and perhaps look more elegant, if the patient can afford it. During the night, the patient is advised to instil a drop of castor oil or liquid paraffin into the affected eye followed by the application of a pad of cotton wool (or a pad soft cloth) and bandage. These simple measures 
will help in preventing the occurrence of exposure keratitis and its disastrous sequelae. By way of medical treatment, in those cases detected soon after the occurrence of paralysis, administration of corticosteriods and antiinflammatory drugs is worthy of trial. In addition, vasodilator drugs such as nicotinic acid in a dose of $100 \mathrm{mg}$ twice a day after food, can be of benefit when given along with injections of vitamin B1, B6 and B12. Physiotherapeutic exercises comprising active forced closure of the lids, massage of the paralysed muscles and electrical stimulation of the viable muscles will considerably aid in the recovery of the protective function in the eye. Recovery of paralytic deformities in leprosy is unpredictable. Medical and physiotherapeutic measures are to be vigorously pursued. When these measures fail surgical procedures will have to be undertaken, depending upon the needs of the patient and the availability of facilities.

In the potentially serious form of eye involvement arising from bacillary invasion of the elements of the eye, institution and uninterrupted continuation of specific treatment in appropriate doses, supplemented by a periodical examination of the eye and protection of the eye from external elements with dark glasses will serve to save the eyes from damage.

Over and above these measures, health education of the patients with regard to the care of the eyes is vital. Many of them are not only ignorant of the potentially serious damage the disease may cause the eyes, but also believe that visual damage sometimes culminating in total loss of vision is a necessary accompaniment of the disease.

\section{Acknowledgements}

Grateful thanks are expressed to the patients who willingly submitted to the examination. Thanks are also due to Sri R. Gopal for secretarial assistance.

\section{References}

Abraham, J. C. (1976). Prevention and treatment of eye complications in leprosy. Lepr. India 4 (Suppl.), 763.

Antia, N. H., Divekar, S. C. and Dastur, D. K. (1966). The facial nerve in leprosy 1. Clinical operative aspects. Int. J. Lepr. 34, 103.

Brand, P. W. (1959). Temperature variations and leprosy deformity. Int. J. Lepr. 27, 1.

Brand, Margaret. (1976). Personal communication.

Jupta, C. P. (1976). Eye complications in leprosy. Lepr. India 4 (Suppl.), 529.

Harrell, J. D. (1977). Ocular leprosy in the Canal Zone. Int. J. Lepr. 45, 56.

World Health. (Feb, March, 1978). 